\title{
Two Higher Order Iterative Methods for Solving Nonlinear Equations
}

\author{
Jivandhar Jnawali ${ }^{1}$, Chet Raj Bhatta ${ }^{2}$ \\ ${ }^{1}$ Department of Mathematics, Ratna Rajyalaxmi Campus, Tribhuvan University, Kathmandu, Nepal \\ ${ }^{2}$ Central Department of Mathematics Tribhuvan University, Kathmandu, Nepal \\ Corresponding author:jnawalij@gmail.com
}

Received: Jan 5, $2018 \quad$ Revised: Jan 20, $2018 \quad$ Accepted: Jan 25, 2018

\begin{abstract}
The main purpose of this paper is to derive two higher order iterative methods for solving nonlinear equations as variants of Mir, Ayub and Rafiq method. These methods are free from higher order derivatives. We obtain these methods by amalgamating Mir, Ayub and Rafiq method with standard secant method and modified secant method given by Amat and Busquier. The order of convergence of new variants are four and six. Also, numerical examples are given to compare the performance of newly introduced methods with the similar existing methods.
\end{abstract}

2010 AMS Subject Classification: 65H05

Keywords: Newton's method, secant method, iterative method, nonlinear equation, order of convergence

\section{Introduction}

Finding the solutions of single variable nonlinear equations efficiently is an important consideration in numerical analysis and has wide range of applications in all areas of science and engineering. Finding analytic solutions of such equations is not always possible and most widely used classical numerical methods for solving such equations are the Newton method [3]

$$
x_{n+1}=x_{n}-\frac{f\left(x_{n}\right)}{f^{\prime}\left(x_{n}\right)}, \quad n \geq 0
$$

and secant method [3]

$$
x_{n+1}=x_{n}-\frac{x_{n}-x_{n-1}}{f\left(x_{n}\right)-f\left(x_{n-1}\right)} f\left(x_{n}\right), n \geq 1
$$

The order of convergence of the Newton method is 2 and the secant method is 1.618. Secant method (2) can be considered as a simplification of Newton's method (1), where $f^{\prime}(x)$ is replaced by 


$$
\frac{f\left(x_{n}\right)-f\left(x_{n-1}\right)}{x_{n}-x_{n-1}}
$$

The main limitation of this method with respect to the Newton method is of order since the above expression is not optimal solution of $f^{\prime}\left(x_{n}\right)$. In order to avoid this problem, Amat and Basquier [2] provide following generalization of secant method:

$$
x_{n+1}=x_{n}-A_{n}^{-1} f\left(x_{n}\right)
$$

where

$$
A_{n}=\left[y_{n}, x_{n} ; f\right]=\frac{f\left(x_{n}\right)-f\left(y_{n}\right)}{x_{n}-y_{n}}, \quad " y_{n}=x_{n}+\delta_{n}\left(x_{n-1}-x_{n}\right) ", \quad \delta_{n} \leq\left|O\left(e_{n}\right)^{\frac{3}{2}}\right| .
$$

The order of convergence of this method is same as Newton's method.

In the last two decades, some researchers have developed several numerical methods based on integral approximations (see $[1,4,6,7,8,9,10,11,13,15,16,17$, and 18]).

In [5], Hassanov, Ivanov and Nedzhibov suggested a new iterative method

$$
\begin{aligned}
& x_{n+1}=x_{n}-\frac{6 f\left(x_{n}\right)}{f^{\prime}\left(x_{n}\right)+4 f^{\prime}\left(\frac{x_{n}+x_{n}^{*}}{2}\right)+f^{\prime}\left(x_{n}^{*}\right)} \\
& \text { where } \quad x_{n}^{*}=x_{n}-\frac{f\left(x_{n}\right)}{f^{\prime}\left(x_{n}\right)}
\end{aligned}
$$

by approximating the integral in Newton's theorem

$$
f(x)=f\left(x_{n}\right)+\int_{x_{n}}^{x} f^{\prime}(\lambda) d \lambda
$$

using the Simpson rule. Combining this method with the quadratically convergent method due to Kanwar, Kukreja and Singh [14],

$$
x_{n+1}=x_{n}-\frac{2 f\left(x_{n}\right)}{f^{\prime}\left(x_{n}\right)+p \sqrt{\left(f^{\prime 2}\left(x_{n}\right)+4 f^{2}\left(x_{n}\right)\right)}}
$$

Mir, Ayub and Rafiq [15] obtained a new third order method

$$
\begin{aligned}
& x_{n+1}=x_{n}-\frac{6 f\left(x_{n}\right)}{f^{\prime}\left(x_{n}\right)+4 f^{\prime}\left(\frac{x_{n+1}^{*}+x_{n}}{2}\right)+f^{\prime}\left(x_{n+1}^{*}\right)} \\
& \text { where } \quad x_{n+1}^{*}=x_{n}-\frac{2 f\left(x_{n}\right)}{f^{\prime}\left(x_{n}\right)+p \sqrt{\left(f^{\prime 2}\left(x_{n}\right)+4 f^{2}\left(x_{n}\right)\right)}}
\end{aligned}
$$

for solving nonlinear equation $f(x)=0$, where $p$ is chosen as positive or negative sign so as to make the denominator largest in magnitude to avoid numerical difficulties. Our aim, in this paper, is to find two variants of method (6) by combining the iteration of this method with secant method and modified secant method. The order of convergence of new methods will be 4 and 6 . 


\section{Description of Methods}

In this section, we propose two variants of Mir, Ayub and Rafiq method (6). At first, we propose a method by performing the iterations of Mir, Ayub and Rafiq method alternately with secant method. The corresponding method becomes

$$
\begin{array}{ll}
x_{n+1} & =\overline{x_{n}}-\frac{\overline{x_{n}}-x_{n}}{f\left(\overline{x_{n}}\right)-f\left(x_{n}\right)} f\left(\overline{x_{n}}\right) \\
\text { where } \quad & \quad \overline{x_{n}}=x_{n}-\frac{6 f\left(x_{n}\right)}{f^{\prime}\left(x_{n}\right)+4 f^{\prime}\left(\frac{x_{n+1}^{*}+x_{n}}{2}\right)+f^{\prime}\left(x_{n+1}^{*}\right)} \\
\text { with } \quad x_{n+1}^{*}=x_{n}-\frac{2 f\left(x_{n}\right)}{f^{\prime}\left(x_{n}\right)+p \sqrt{\left(f^{\prime 2}\left(x_{n}\right)+4 f^{2}\left(x_{n}\right)\right)}}
\end{array}
$$

In this method, $p$ is chosen as positive or negative sign so as to make the denominator largest in magnitude to avoid numerical difficulties.

Next, the iterations of Mir, Ayub and Rafiq method (6) are performed alternately with modified secant method (3). Then the corresponding method becomes

$$
\begin{aligned}
x_{n+1}^{*} & =x_{n}-\frac{2 f\left(x_{n}\right)}{f^{\prime}\left(x_{n}\right)+p \sqrt{\left(f^{\prime 2}\left(x_{n}\right)+4 f^{2}\left(x_{n}\right)\right)}} \\
\overline{x_{n}} & =x_{n}-\frac{6 f\left(x_{n}\right)}{f^{\prime}\left(x_{n}\right)+4 f^{\prime}\left(\frac{x_{n+1}^{*}+x_{n}}{2}\right)+f^{\prime}\left(x_{n+1}^{*}\right)} \\
x_{n+1} & =\overline{x_{n}}-A_{n}^{-1} f\left(\overline{x_{n}}\right) \\
\text { where } \quad A_{n} & =\left[y_{n}, \overline{x_{n}} ; f\right]=\frac{f\left(\overline{x_{n}}\right)-f\left(y_{n}\right)}{\overline{x_{n}}-y_{n}} \\
\text { with } \quad y_{n} & =\overline{x_{n}}+\delta_{n}\left(x_{n}-\overline{x_{n}}\right), \quad \delta_{n} \leq\left|O\left(e_{n}\right)^{\frac{3}{2}}\right| .
\end{aligned}
$$

\section{Analysis of Convergence}

For the convergence analysis of method (7) - (9), we prove following result:

Theorem 1. Let $f$ be a function having sufficient number of derivatives in a neighborhood of $\alpha$, which is a simple root of equation $f(x)=0$. Then, the iterative method (7)-(9) is of order 4 for $p=1$. 
Proof. Let $e_{n}$ and $\overline{e_{n}}$ be the errors in $x_{n}$ and $\overline{x_{n}}$, respectively, that is, $x_{n}=\alpha+e_{n}$ and $\overline{x_{n}}=\alpha+\overline{e_{n}}$. Denote $c_{k}=\frac{f^{k}(\alpha)}{k ! f^{\prime}(\alpha)}, k=2,3,4 \ldots$. It was shown by Mir, Ayub and Rafiq in [15] that the error equation of (8) is given by

$$
\overline{e_{n}}=c_{2}^{2} e_{n}^{3}+O\left(e_{n}^{4}\right)
$$

Here,

$$
\begin{aligned}
\overline{x_{n}}-x_{n} & =\left(\alpha+\overline{e_{n}}\right)-\left(\alpha+e_{n}\right) \\
& =\overline{e_{n}}-e_{n} \\
& =c_{2}^{2} e_{n}^{3}-e_{n}+O\left(e_{n}^{4}\right) .
\end{aligned}
$$

By Taylor's expansion, we get

$$
\begin{aligned}
f\left(x_{n}\right) & =f\left(\alpha+e_{n}\right) \\
& =f^{\prime}(\alpha)\left[e_{n}+c_{2} e_{n}^{2}+c_{3} e_{n}^{3}+O\left(e_{n}^{4}\right)\right]
\end{aligned}
$$

and using (11), we obtain

$$
\begin{aligned}
f\left(\overline{x_{n}}\right)= & f\left(\alpha+\overline{e_{n}}\right) \\
= & f^{\prime}(\alpha)\left[c_{2}^{2} e_{n}^{3}+O\left(e_{n}^{6}\right)\right]
\end{aligned}
$$

Thus, we get

$$
\begin{aligned}
f\left(\overline{x_{n}}\right)-f\left(x_{n}\right) & =f^{\prime}(\alpha)\left[c_{2}^{2} e_{n}^{3}+O\left(e_{n}^{6}\right)\right]-f^{\prime}(\alpha)\left[e_{n}+c_{2} e_{n}^{2}+c_{3} e_{n}^{3}+O\left(e_{n}^{4}\right)\right] \\
& =-f^{\prime}(\alpha) e_{n}\left[1+c_{2} e_{n}+\left(c_{3}-c_{2}^{2}\right) e_{n}^{2}+O\left(e_{n}^{3}\right)\right]
\end{aligned}
$$

and

$$
\begin{aligned}
\frac{\left.\overline{\left(x_{n}\right.}-x_{n}\right) f\left(\overline{x_{n}}\right)}{f\left(\overline{x_{n}}\right)-f\left(x_{n}\right)}= & \frac{\left[c_{2}^{2} e_{n}^{3}-e_{n}+O\left(e_{n}^{4}\right)\right] f^{\prime}(\alpha)\left[c_{2}^{2} e_{n}^{3}+O\left(e_{n}^{6}\right)\right]}{-f^{\prime}(\alpha) e_{n}\left[1+c_{2} e_{n}+\left(c_{3}-c_{2}^{2}\right) e_{n}^{2}+O\left(e_{n}^{3}\right)\right]} \\
& =\left[c_{2}^{2} e_{n}^{3}+O\left(e_{n}^{5}\right)\right]\left[1+c_{2} e_{n}+\left(c_{3}-c_{2}^{2}\right) e_{n}^{2}+O\left(e_{n}^{3}\right)\right]^{-1} \\
& =c_{2}^{2} e_{n}^{3}-c_{2}^{3} e_{n}^{4}+O\left(e_{n}^{5}\right)
\end{aligned}
$$

Thus, the error equation in (7) is given by

$$
\begin{aligned}
e_{n+1} & =\overline{e_{n}}-c_{2}^{2} e_{n}^{3}+c_{2}^{3} e_{n}^{4}+O\left(e_{n}^{5}\right) \\
& =c_{2}^{2} e_{n}^{3}+O\left(e_{n}^{4}\right)-c_{2}^{2} e_{n}^{3}+c_{2}^{3} e_{n}^{4}+O\left(e_{n}^{5}\right) \\
& =A e_{n}^{4}+O\left(e_{n}^{5}\right),
\end{aligned}
$$

where A is some constant. The assertion is, therefore, proved.

For the convergence analysis of method (10), we prove following result:

Theorem 2. Let $f$ be a function having sufficient number of derivatives in a neighborhood of $\alpha$, which is a simple root of equation $f(x)=0$. Then, the iterative method (10) is of order at least 6 for $p=1$ and suitable choice of $\delta$. 
Proof. We prove this theorem in the line of proof of Theorem 1. The error $\overline{e_{n}}$ in $\overline{x_{n}}$ is given by

$$
\overline{e_{n}}=c_{2}^{2} e_{n}^{3}+O\left(e_{n}^{4}\right)
$$

Since

$\delta_{n}\left(x_{n}-\overline{x_{n}}\right)$,

$$
y_{n}=\overline{x_{n}}+\delta_{n}\left(x_{n}-\overline{x_{n}}\right)=\overline{x_{n}}+a_{n} \text {, where } a_{n}=
$$

by using Taylor expansion, we get

$$
f\left(y_{n}\right)=f\left(\overline{x_{n}}+a_{n}\right)=f\left(\overline{x_{n}}\right)+a_{n} f^{\prime}\left(\overline{x_{n}}\right)+\frac{a_{n}^{2}}{2} f^{\prime \prime}\left(\overline{x_{n}}\right)+\cdots .
$$

Also,

$$
\begin{aligned}
A_{n} & =\frac{f\left(\overline{x_{n}}\right)-f\left(y_{n}\right)}{\overline{x_{n}}-y_{n}} \\
& =\frac{a_{n} f^{\prime}\left(\overline{x_{n}}\right)+\frac{a_{n}^{2}}{2} f^{\prime \prime}\left(\overline{x_{n}}\right)+\cdots}{a_{n}} \\
& =f^{\prime}\left(\overline{x_{n}}\right)+\frac{a_{n}}{2} f^{\prime \prime}\left(\overline{x_{n}}\right)+\cdots \\
& =f^{\prime}\left(\overline{x_{n}}\right)+O\left(a_{n}\right) .
\end{aligned}
$$

Thus, from (10), we have

$$
\begin{aligned}
x_{n+1} & =\overline{x_{n}}-\frac{f\left(\overline{x_{n}}\right)}{A_{n}} \\
& =\overline{x_{n}}-\frac{f\left(\overline{x_{n}}\right)}{f^{\prime}\left(\overline{x_{n}}\right)+O\left(a_{n}\right)} \\
& =\overline{x_{n}}-\frac{f\left(\overline{x_{n}}\right)}{f^{\prime}\left(\overline{x_{n}}\right)}\left[1+O\left(a_{n}\right)\right]^{-1} \\
& =\overline{x_{n}}-\frac{f\left(\overline{x_{n}}\right)}{f^{\prime}\left(\overline{x_{n}}\right)}\left[1+O\left(a_{n}\right)+O\left(a_{n}^{2}\right)+\cdots\right. \\
& =\overline{x_{n}}-\frac{f\left(\overline{x_{n}}\right)}{f^{\prime}\left(\overline{x_{n}}\right)}+O\left(a_{n}\right) .
\end{aligned}
$$

Thus, the method (10) can be written as

$$
x_{n+1}=\overline{x_{n}}-\frac{f\left(\overline{x_{n}}\right)}{f^{\prime}\left(\overline{x_{n}}\right)}+O\left(\delta_{n}\left|x_{n}-\overline{x_{n}}\right|\right)
$$

From (11), we have

$$
\begin{aligned}
e_{n+1}= & \overline{e_{n}}-\frac{f\left(\alpha+\overline{e_{n}}\right)}{f^{\prime}\left(\alpha+\overline{e_{n}}\right)}+O\left(\delta_{n}\left|\alpha+e_{n}-\alpha-\overline{e_{n}}\right|\right) \\
= & \overline{e_{n}}-\left[\overline{e_{n}}+c_{2}{\overline{e_{n}}}^{2}+c_{3}{\overline{e_{n}}}^{3}+O\left({\overline{e_{n}}}^{4}\right)\right]\left[1+2 c_{2} \overline{e_{n}}+3 c_{3}{\overline{e_{n}}}^{2}+O\left({\overline{e_{n}}}^{3}\right)\right]^{-1} \\
& +O\left(\delta_{n}\left|e_{n}-\overline{e_{n}}\right|\right)
\end{aligned}
$$




$$
\begin{aligned}
& =\overline{e_{n}}-\left[\overline{e_{n}}+c_{2}{\overline{e_{n}}}^{2}-2 c_{3}{\overline{e_{n}}}^{2}+O\left({\overline{e_{n}}}^{3}\right)\right]+O\left(\delta_{n}\left|e_{n}-\overline{e_{n}}\right|\right) \\
& =c_{2}{\overline{e_{n}}}^{2}+O\left({\overline{e_{n}}}^{3}\right)+O\left(\delta_{n}\left|e_{n}-\overline{e_{n}}\right|\right) \\
& =c_{2}\left(c_{2}^{2} e_{n}^{3}\right)^{2}+O\left(\delta_{n}\left|e_{n}-c_{2}^{2} e_{n}^{3}\right|\right)+\cdots \\
& =c_{2}^{5} e_{n}^{6}+O\left(\delta_{n}\left|e_{n}-c_{2}^{2} e_{n}^{3}\right|\right)+\cdots
\end{aligned}
$$

Since $\delta_{n} \leq\left|O\left(\overline{e_{n}}\right)^{\frac{3}{2}}\right|$, so if we assume that $\delta_{n} \leq\left|O\left(\overline{e_{n}}\right)^{2}\right|$, that is, $\delta_{n} \leq\left|O\left(e_{n}\right)^{6}\right|$, then the order of the method (10) is at least 6 .

Remark 1. In practice, we do not have any information about the solution. In this situation, we use the same strategy as the Amat and Basquier indicated in [2] to obtain $\delta_{n}$. The possible strategy to obtain $\left\{\delta_{n}\right\}$ can be

$$
\begin{aligned}
& \delta_{0}=O\left(10^{-k}\right) \leq O\left(e_{n}\right)^{\frac{3}{2}} \\
& \delta_{n}=O\left(\delta_{0}^{2^{n}}\right),
\end{aligned}
$$

where $\mathrm{k}$ is an integer such that

$$
O\left(10^{-k}\right) \leq\left|f(\alpha)-f\left(x_{0}\right)\right| \leq\left|f\left(x_{0}\right)\right| \leq O\left(\left|\alpha-x_{0}\right|\right) .
$$

\section{Numerical Examples}

In this section, we shall compare the performance of newly introduced methods (7) and (10) with the some existing methods. To avoid the numerical difficulties in newly introduced methods, we take $p$ equal to 1 or -1 so as to make the denominator largest in magnitude. For the comparison, we use Matlab Software and stopping criteria $\left|x_{n+1}-x_{n}\right|<(10)^{-12}$ or $\left|f\left(x_{n+1}\right)\right|<(10)^{-14}$ for the iterative process of our results.

Example 1. We apply methods (7), and (10) on the nonlinear equation

$$
x^{6}-x-1=0
$$

From the intermediate value theorem, it is clear that one of the simple roots of (13) lies in the interval $(1,2)$. Taking initial approximation as $x_{0}=1$, Table 1 shows the iterations of the Newton method, Mir, Ayub and Rafiq method (6), and introducing methods (7) and (10). 
Table 1: Comparison of different methods.

\begin{tabular}{|l|l|c|c|c|}
\hline Method & $\mathrm{n}$ & $x_{n}$ & $\left|x_{n}-x_{n-1}\right|$ & $\left|f\left(x_{n}\right)\right|$ \\
\hline Newton's & 1 & 1.200000000000000 & 0.200000000000000 & 0.785983999999999 \\
method & 2 & 1.143575842503044 & 0.056424157496956 & 0.093031957363097 \\
& 3 & 1.134909462242086 & 0.008666380260958 & 0.001907397172335 \\
& 4 & 1.134724221386558 & 0.000185240855528 & 0.000000853719439 \\
& 5 & 1.134724138401536 & 0.000000082985022 & 0.000000000000171 \\
\hline Mir, Ayub & 1 & 1.134724138401519 & 0.000000000000017 & 0.000000000000001 \\
and & 2 & 1.114331079664285 & 0.114331079664285 & 0.199698426927704 \\
Rafiq & 3 & 1.134724138400651 & 0.000052968316718 & 0.000000000008935 \\
method & 4 & 1.134724138401519 & 0.000000000000868 & 0.000000000000001 \\
(p=1) & & & & \\
\hline Present & 1 & 1.142859996170415 & 0.142859996170415 & 0.085360616958645 \\
method (7) & 2 & 1.134724198032685 & 0.008135798137730 & 0.000000613463385 \\
& 3 & 1.134724138401519 & 0.000000059631166 & 0.000000000000001 \\
\hline Present & 1 & 1.134888830552744 & 0.134888830552744 & 0.001694966368938 \\
method (10) & 2 & 1.134724138401519 & 0.000164692151225 & 0.000000000000003 \\
$\left(\delta_{0}=0.15\right)$ & & & & \\
\hline
\end{tabular}

Example 2. We apply methods (7) and (10) on the nonlinear equation

$$
\sin ^{2} x-x^{2}+1=0
$$

From the intermediate value theorem, it is clear that one of the simple roots of (14) lies in the interval $(1,2)$. Taking initial approximation as $x_{0}=2$, Table 2 shows the iterations of the Kanwar, Kukreja, and Singh method (5), Mir, Ayub and Rafiq method (6), and introducing methods (7) and (10) when we apply on (14). 
Table 2: Comparison of different methods.

\begin{tabular}{|l|l|c|c|c|}
\hline Method & $\mathrm{n}$ & $x_{n}$ & $\left|x_{n}-x_{n-1}\right|$ & $\left|f\left(x_{n}\right)\right|$ \\
\hline Kunar, & 1 & 1.611941042164389 & 0.388058957835611 & 0.600045855942974 \\
Kukraia & 2 & 1.436057898886696 & 0.175883143277692 & 0.080307137216431 \\
and Singh & 3 & 1.405269547988669 & 0.030788350898027 & 0.001932292231027 \\
method & 4 & 1.404492122350878 & 0.000777425637791 & 0.000001177029015 \\
& 5 & 1.404491648215517 & 0.000000474135360 & 0.000000000000437 \\
& 6 & 1.404491648215341 & 0.000000000000176 & 0.000000000000000 \\
\hline Mir, Ayub and & 1 & 1.463929732530918 & 0.536070267469082 & 0.154467321201545 \\
Rafiq method & 2 & 1.404614185557379 & 0.059315546973539 & 0.000304224828280 \\
(p=1) & 3 & 1.404491648216471 & 0.000122537340908 & 0.000000000002804 \\
& 4 & 1.404491648215341 & 0.000000000001130 & 0.000000000000000 \\
\hline Present & 1 & 1.422910813234791 & 0.577089186765209 & 0.046386337604170 \\
method (7) & 2 & 1.404491702133259 & 0.018419111101532 & 0.000000133849771 \\
& 3 & 1.404491648215341 & 0.000000053917918 & 0.000000000000000 \\
\hline Present & 1 & 1.404751654708388 & 0.595248345291612 & 0.000645590555316 \\
method (10) & 2 & 1.404491648215341 & 0.000260006493047 & 0.000000000000000 \\
$\left(\delta_{0}=0.1\right)$ & & & & \\
\hline
\end{tabular}

\section{Conclusion}

From the above comparison tables, we observe that newly introduced methods are easily compete with existing Newton's method, Kanwar, Kukreja, and Singh's method (5) and Mir, Ayub and Rafiq's method (6). For the suitable choice of the values of $p$ and $\delta_{0}$, newly introduced methods (7) and (10) provide better results.

\section{References}

[1] Ababneh OY (2012), New Newton's method with third-order convergence for solving nonlinear equations, World Academy of Science and Engineering and Technology, 61 : 10711073.

[2] Amat S and Busquier S (2003), On a higher order secant method, Applied Mathematics and Computation, 141: 321-329.

[3] Burdan LR and Fairs JD (2011), Numerical Analysis, Cengage Learning, 2011.

[4] Dheghain M and Hajarian M (2011), New iterative method for solving nonlinear equations with Fourth-order convergence, International Journal of Computer Mathematics, 87 : 834-839.

[5] Hassanov VI, Ivanov IG and Nedzhibov G (2002), A new modification of Newton's method, Application of Mathematics in Engineering, 27 : 278-286. 
[6] Homeier HHH (2005), On Newton's type methods with cubic convergence, Journal of Computational and Applied Mathematics, 176 (2) : 425- 432.

[7] Jain D (2013), Families of Newton-like methods with fourth-order convergence, International Journal of Computer Mathematics, 90 : 1072-1082.

[8] Jain P, Bhatta CR and Jnawali J (2016), Newton type iterative methods with higher order of Convergence, Journal of Numerical Analysis and Approximation Theory, 45 : 14-26.

[9] Jayakumar J and Kalyanasundram M (2013), Modified Newton's method using harmonic mean for solving nonlinear equations, IOSR Journal of Mathematics, 7 : 93-97.

[10] Jayakumar J and Kalyanasundram M (2013), Generalized power means modification of Newton's method for simple roots of nonlinear equation, International Journal of Pure and Applied sciences and Technology, $18: 45-51$.

[11] Jayakumar J and Kalyansundram M (2015), Power means based modification of Newton's method for solving nonlinear equations with cubic convergence, International Journal of Applied Mathematics and Computation, 6 : 1-6.

[12] Jisheing K, Yitian L and Xiuhua W (2007), Third order modification of Newton's method, Journal of Computational and Applied Mathematics, 205 : 1-5.

[13] Jnawali $\mathrm{J}$ and Bhatta CR, A new variant of Newton's method with fourth-order convergent, Journal of Institute of Science and Technology, 21 : 86-89.

[14] Kanwar MV, Kukreja VK and Singh S (2005), On a class of quadratically convergent iteration formulae, Applied mathematics and Computation, 166 : 633-637.

[15] Mir NA, Ayub K and Rafiq (2010), A third-order convergent iterative method for solving nonlinear equations, International Journal of Computer Mathematics, 4 : 849-854.

[16] Özban AY (2004), Some new variants of Newton's method, Applied Mathematics Letters, 17: 677-682.

[17] Wang P (2011), A third order family of Newton like iteration method for solving nonlinear equation, Journal of Numerical Mathematics and Stochastics, 3(1) : 11-19.

[18] Weerakoon S and Fernando TGI (2002), A variant of Newton's method with accelerated thirdorder convergence, Applied Mathematics Letters, 13 : 87-93. 